Biokatalyse

\title{
N-Hydroxydiamine - vielseitige Bausteine für Wirkstoffsynthesen
}

\author{
ARTUR MAIER, SARAH WANSEL, ALVARO GOMEZ BARAIBAR, CAROLIN MÜGGE, \\ DIRK TISCHLER \\ AG MIKROBIELLE BIOTECHNOLOGIE, RUHR-UNIVERSITÄT BOCHUM
}

\section{An enzymatic cascade from $\omega$-amino amino acids towards monohy- droxylated $\alpha, \omega$-diamines gives highly interesting materials for process- ing into fine chemicals. With two simple UV-vis spectroscopy-based screening methods, enzyme candidates with a compatibility window to perform a three-enzyme cascade from lysine and ornithine to the desired products were identified. The results give first insight into the feasibility of the desired cascade and put handles on future reaction engineering tasks.}

DOI: $10.1007 / \mathrm{s} 12268-020-1374-6$

(C) Die Autoren 2020

Aminosäuren mit einer $\omega$-Aminoseitenkette, wie Ornithin (Orn) und Lysin (Lys), stellen Rohstoffquellen für funktionalisierte aliphatische Diamine dar. Durch Decarboxylierung können die Diamine Putrescin (Put, 1,4-Butandiamin) aus Ornithin und Cadaverin (Cad, 1,5-Pentandiamin) aus Lysin generiert werden [1]. Aminosäure-Decarboxylasen können hierbei ausgezeichnete Ausbeuten erwirken. Eine selektive Monofunktionalisierung von nur einer Aminogruppe im Anschluss stellt eine weit größere Herausforderung dar. Mit einer N-hydroxylierenden Monooxygenase (NMO) ist es z. B. möglich, eine der beiden terminalen Aminofunktionen zu hydroxylieren [2] und so eine Grundchemikalie für weitere Anwendungen, etwa der Herstellung von Hydroxytriazenen mit biologischer Aktivität, zu erhalten [3].

Die hohe Substratselektivität und eine geringe Aktivität der genannten Enzyme waren bisher Hürden für Anwendungen. Actinobakterien setzen NMOs in ihrer Siderophor-Biosynthese ein, die eine gewisse Promiskuität im Substratspektrum bei vergleichsweise hohen Aktivitäten aufzeigen [2]. Diese Enzymklasse wollten wir nutzbar machen und erstmals den Teil der Sidero-

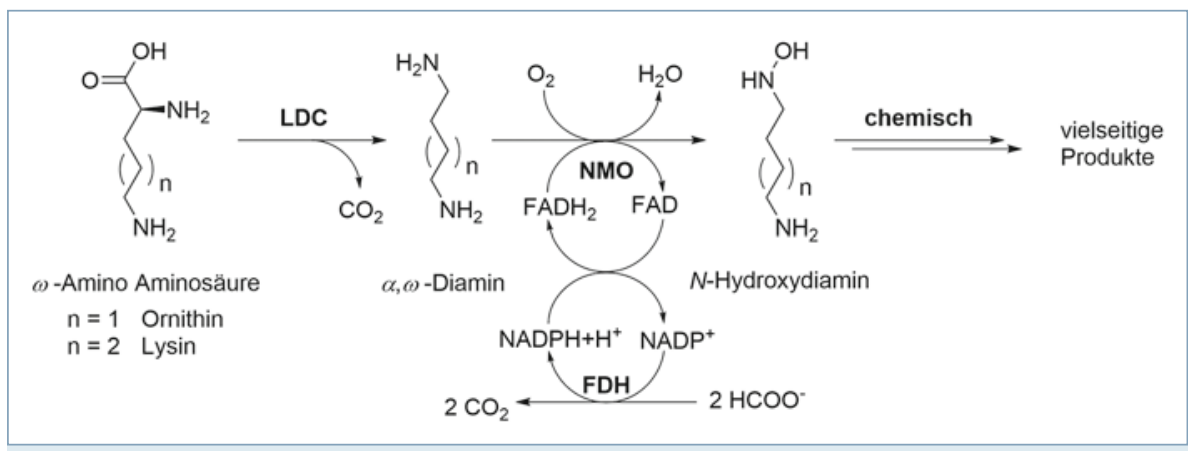

$\Delta$ Abb. 1: Enzymkaskade zu Herstellung von N-Hydroxydiaminen. Ornithin und Lysin können mit Lysindecarboxylasen (LDCs) und N-hydroxylierenden Monooxygenasen (NMOs) über $\alpha, \omega$-Diamine zu N-Hydroxydiaminen umgesetzt werden. Die NMO ist FAD-abhängig, was mithilfe von NADPH regeneriert wird. Letzteres wird durch Formiatdehydrogenase (FDH) unter Verbrauch von Formiat bereitgestellt.
phor-Biosynthese als semi-natürliche Kaskade einsetzen. Unser Ziel war es, das Potenzial von NMOs im Zusammenspiel von Kofaktorregeneration und weiteren Enzymen zu erforschen und erste Schritte in Richtung ihrer biotechnologischen Nutzung zu gehen. Es sollten die Rahmenbedingungen für erfolgreiche N-Hydroxylierungen abgesteckt und geeignete Systeme für die Substratbereitstellung mittels Aminosäure-Decarboxylasen sowie zur Regeneration von Kofaktoren gefunden werden.

$\mathrm{N}$-Hydroxylierung: $\mathrm{N}$-Hydroxylase und NADPH-Regeneration

NMOs (EC 1.14.13.B10) [4] gehören zu den Flavin-abhängigen Enzymen mit einem engen Substratspektrum [5]. Sie spielen eine Rolle in der Siderophor-Synthese von Bakterien und Pilzen sowie in der Immunantwort von Pflanzen [6]. Traditionell setzen sie Aminosäuren wie Lysin und Ornithin, aber auch die Diamine Cadaverin und Putrescin, mit Sauerstoff um. Die für die Reaktion benötigten Redoxäquivalente werden durch Nikotinamidadenindinukleotide $(\mathrm{NAD}(\mathrm{P}) \mathrm{H})$ bereitgestellt. Mithilfe von NAD(P)H reduziert die NMO Flavinadenindinukleotid (FAD), so erzeugtes $\mathrm{FADH}_{2}$ kann zum Umsatz des terminalen Amins mit Sauerstoff unter Generierung von Wasser verwendet werden. Aus vorläufigen Studien zur Aktivität zweier NMOs - TheA aus Thermocrispum agreste [7] und GorA aus Gordonia rubripertincta [2] wurde GorA als geeigneter Katalysator für die Umwandlung von Putrescin in N-Hydroxyputrescin identifiziert. Das Enzym konnte durch rekombinante Expression aus Escherichia coli und Aufreinigung über NickelAffinitätschromatographie gewonnen werden.

Für biotechnologische Ansätze muss ein Regenerationssystem für das NADPH/ $\mathrm{NADP}^{+}$-Redoxpaar eingesetzt werden, das mit den anderen Schritten der Kaskade kompatibel ist. Formiatdehydrogenase (FDH) ist grundsätzlich geeignet für die Bereitstellung von reduziertem NADH zur Aktivierung von FAD (Abb. 1). In unseren Studien nutzten wir nun eine Variante der FDH aus Candida 


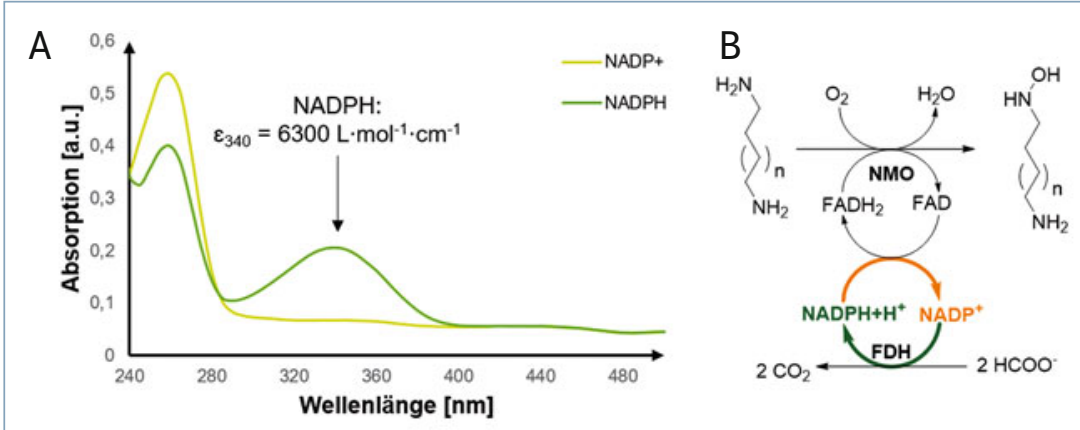

D

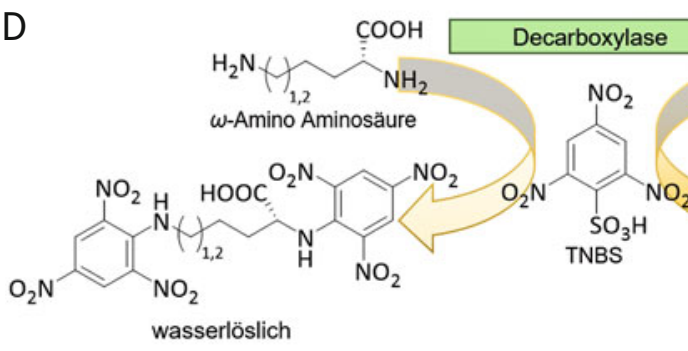

$>\mathrm{H}_{2} \mathrm{~N}+\mathrm{N}_{1,2} \mathrm{NH}_{2}$

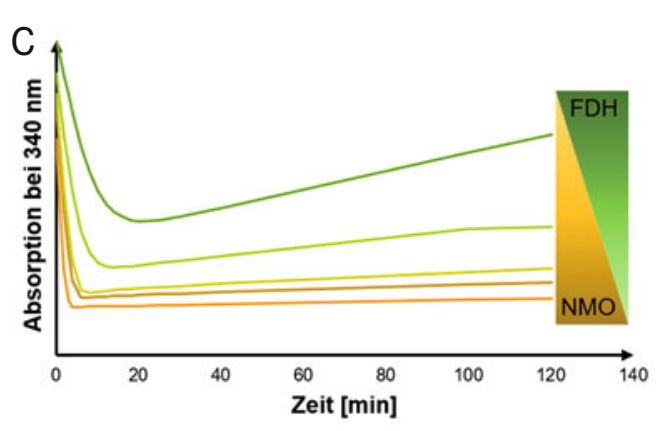

E

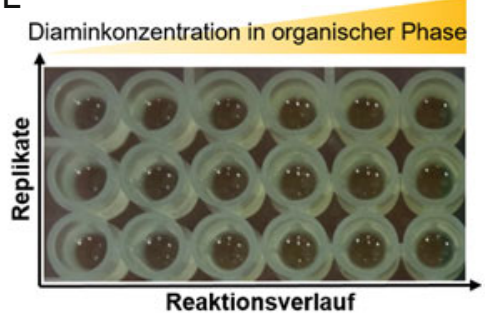

$\triangle$ Abb. 2: UV-Vis-basierte Methoden für die Kaskadenentwicklung. A-C, NADPH-Bildung. NADP ${ }^{+}$und NADPH haben definierte UV-Vis-Spektren (A). Die Konzentration von NADPH kann bei $340 \mathrm{~nm}$ in operando verfolgt werden (B, C). NMO: N-hydroxylierende Monooxygenase; FDH: Formiatdehydrogenase. D, E, TNBS-Test. Reaktion der zwei Analyten Aminosäure und Diamin mit 2,4,6-Trinitrobenzolsulfonsäure (TNBS; D) und Verfolgen der Diaminbildung in der organischen Phase (E).

boidinii (CboFDH D1950/Y196H) [8, 9]. Die Wildtyp-CboFDH ist inaktiv gegenüber $\mathrm{NADP}^{+}$, die hier genutzte Variante kann hingegen beide Nicotinamid-Kofaktoren umsetzen. Auch wenn die FDH-Variante weniger aktiv gegenüber $\mathrm{NADP}^{+}$als gegenüber $\mathrm{NAD}^{+}$ ist (spezifische Aktivitäten von $0,64 \pm 0,03$ $\mathrm{U} \cdot \mathrm{mg}^{-1}$ versus $1,03 \pm 0,05 \mathrm{U} \cdot \mathrm{mg}^{-1}$ ), kann hiermit ein hinreichend effizientes Regenerationssystem für die gewünschte Anwendung bereitgestellt werden.

Zur Etablierung der N-Hydroxylierung von Putrescin zu N-Hydroxyputrescin wurde ein einfach durchzuführendes Testsystem genutzt. Initiale Aktivitätsbestimmungen wurden durchgeführt, indem die Konzentration von NADPH spektrophotometrisch bei 340 Nanometer verfolgt wurde: Für die NMO-Teilreaktion wurde die Abnahme, für die FDH-Teilreaktion die Zunahme an NADPH in der Reaktionslösung beobachtet (Abb. 2A-C). Zunächst wurden verschiedene Reaktionsbedingungen für beide Enzyme unabhängig untersucht. Besonderes Augenmerk lag auf der Kompatibilität der Bedingungen für GorA und die CboFDH-Variante. Hierfür wurde der Einfluss des am pH-Optimum von GorA (pH 8) eingesetzten Puffers sowie der Zusatz von Salzen als Stabilisatoren variiert (Abb. 3A, B). Als geeignete Kaskadenbedingungen wurden $50 \mathrm{mM}$ HEPES-Puffer ( $\mathrm{pH} \mathrm{8,0)} \mathrm{mit} \mathrm{einem} \mathrm{erhöhten}$ Salzgehalt (50 mM NaCl und 125 mM Formiat) gefunden. Um im GorA/CboFDH-System eine möglichst effiziente Bereitstellung von $\mathrm{FADH}_{2}$ zu gewährleisten, wird CboFDH im Überschuss eingesetzt. Im zeitlichen Verlauf kann so nach einer anfänglichen Nettoreduktion von zugefügtem NADPH (Sättigung von GorA mit Substrat) eine graduelle Anreicherung des reduzierten Kofaktors erreicht und das kontinuierliche Betreiben der Hydroxylierungsreaktion sichergestellt werden. Der entsprechende charakteristische Verlauf des NADPH-Tests wies auf eine intakte Enzymkaskade hin (Abb. 2C: typischer Reaktionsverlauf). Weiterhin wurde die Bildung von N-Hydroxyputrescin mithilfe von Flüssigchromatographie/ Massenspektrometrie(LC/MS)-Analytik nachgewiesen.

\section{Decarboxylierung: Diamine durch Lysindecarboxylasen}

Um Substrate für die N-Hydroxylierung bereitzustellen, wurden vier bekannte Lysindecarboxylasen (LDCs; EC 4.1.1.18) auf ihre Aktivität gegenüber Lysin und Ornithin untersucht: DAD_Ps aus Pimelobacter simplex, GorB aus G. rubripertincta CWB2, CadA aus Escherichia coli K12 AG1 und $L d c$ C aus E. coli BL21(DE3). Alle Enzyme konnten in $E$. coli produziert und nach Nickel-Affinitätschromatographie erfolgreich isoliert werden.

Für eine einfache und schnelle Bestimmung der Decarboxylierungsaktivität wurde ein kolorimetrischer Test mit 2,4,6-Trinitrobenzolsulfonsäure (TNBS) im 96-Well-Format etabliert (Abb. 2C, D, [10, 11]). Dank der unterschiedlichen Löslichkeit von TNB-Aminosäure- und TNB-Diamin-Addukten in Toluol lassen sich die intensiv gelb gefärbten TNB-Diamin-Addukte photometrisch in Toluol nachweisen, während TNB-AminosäureAddukte wasserlöslich sind.

Basierend auf initialen Produktbildungsraten gegenüber Lysin wurde CadA für die Kaskade gewählt, da es unter Standardbedingungen die höchste Umsatzrate (135 U/mg) zeigte. Um die Decarboxylierung in der Enzymkaskade ablaufen zu lassen, wurde die Aktivität von CadA unter verschiedenen Reaktionsbedingungen untersucht (Abb. 3C). Die Variation von Medium und Salzkonzentration brachte eine Reduzierung der Enzymleistung mit sich, trotzdem konnten Aktivitäten von 48 U/mg unter Kaskadenbedingungen erreicht werden.

\section{Drei-Enzym-Kaskade: von der Aminosäure zum N-Hydroxydiamin}

Schließlich wurden erste Reaktionen im Kaskadenansatz realisiert (Abb. 3D). Aufgrund der vorher für alle Teilschritte gefundenen Bedingungen erwies es sich als sinnvoll, die zwei enzymatischen Schritte sequenziell zu betreiben. Auf diese Weise konnte der für die Decarboxylierung nachteilige, für die 

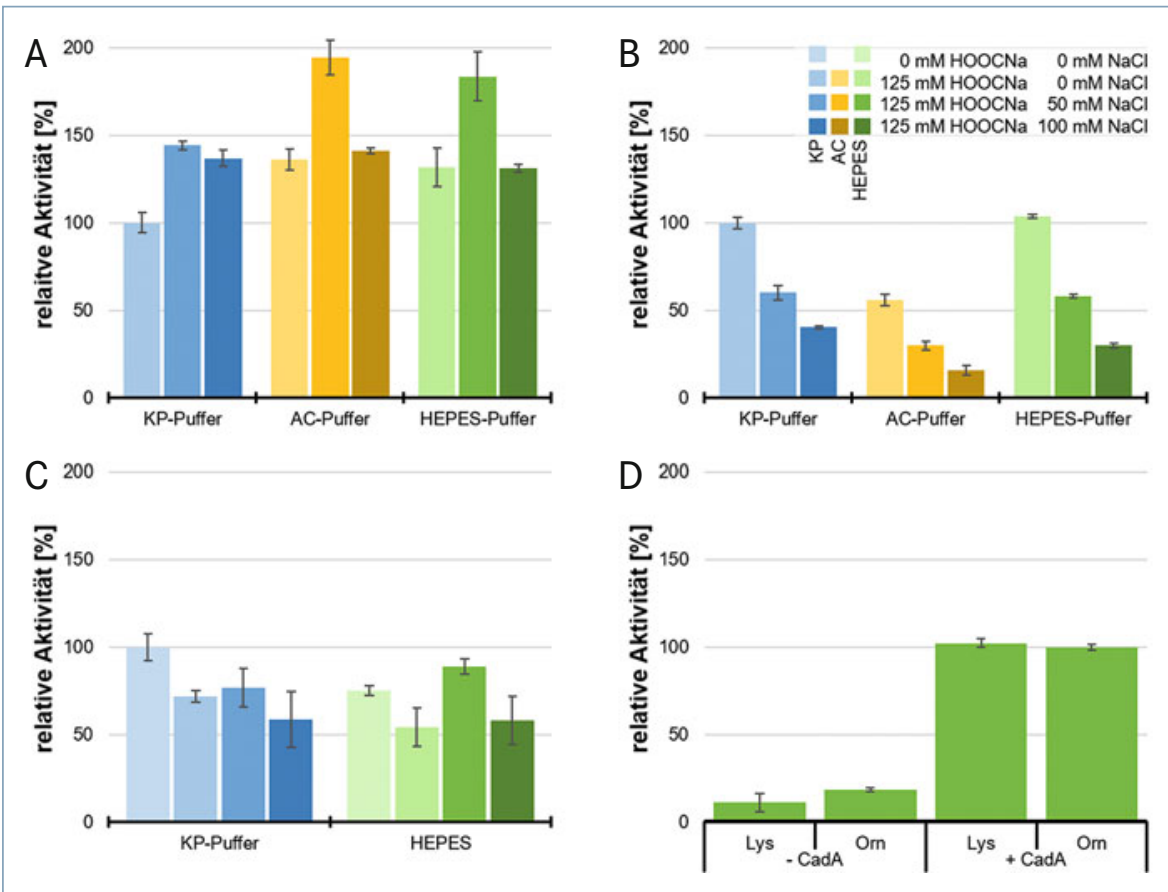

$\Delta$ Abb. 3: Spezifische Aktivität der Enzymsysteme. A, Aktivität der N-hydroxylierenden Monooxygenase (NMO): GorA. B, Formiatdehydrogenase(FDH)-Aktivität: $C b o F D H$, beides mit NADPHAssay. C, Lysindecarboxylase(LDC)-Aktivität: CadA mit TNBS-Test (TNBS: 2,4,6-Trinitrobenzolsulfonsäure). D, Aktivität der Enzyme unter Kaskadenbedingungen (HEPES-Puffer, 50 mM, pH 8; $50 \mathrm{mM} \mathrm{NaCl} ; 125 \mathrm{mM}$ Formiat; $10 \mathrm{mM}$ Aminosäure). Puffer: Kaliumphosphat (KP), Ammoniumcarbonat (AC) und HEPES, Salze: Natriumformiat ( $\mathrm{HCOONa}$ ) und $\mathrm{NaCl}$.

Hydroxylierung aber nötige hohe Salzgehalt im ersten Teil vermieden und eine effiziente Bereitstellung des Intermediats Putrescin gewährleistet werden. Durch Verfolgen des NADPH-Gehalts in der Reaktion wurde die Enzymaktivität nachgewiesen. Kontrollen ohne CadA schlossen ein falsch-positives Ergebnis aufgrund von Aminosäurehydroxylierung oder Entkopplung der NADPH-Bildung durch Anwesenheit der Decarboxylase aus.

\section{Nächste Schritte zu Feinchemikalien}

Die von uns im Enzymkaskadenansatz hergestellten hydroxylierten Diamine können in weiteren Schritten zu wertvollen Feinchemi- kalien funktionalisiert werden. Erste Versuche zur chemischen Kopplung eines Diazoniumsalzes führten erfolgreich zu Hydroxytriazenen und eröffnen eine weite Bandbreite an Möglichkeiten, N-Hydroxydiamine als wertvolle Ausgangsstoffe in verschiedenen Wertschöpfungsketten zu nutzen, z. B. für Komponenten mit antimikrobieller Wirkung.

\section{Danksagung}

Wir danken der DECHEMA (Max-BuchnerStipendium MBFSt 3646). Das Projekt wird durch das Ministerium für Innovation, Wissenschaft und Forschung des Landes Nordrhein-Westfalen (PtJ-TRI/1141ng006) gefördert.

\section{Literatur}

[1] Wendisch VF, Mindt M, Pérez-García F (2018) Biotechnological production of mono- and diamines using bacteria: recent progress, applications, and perspectives. Appl Microbiol Biotechnol 102:3583-3594 [2] Esuola CO, Babalola OO, Heine T et al. (2016) Identification and characterization of a FAD-dependent putrescine N-hydroxylase (GorA) from Gordonia rubripertincta CWB2. J Mol Catal B Enzym 134:378-389 [3] Goswami AK, Purohit DN (2002) Synthesis and antimicrobial activities of some hydroxytriazenes: a new class of biologically active compounds. Anal Sci 17icas:i789-i791 [4] Huijbers MME, Montersino S, Westphal AH et al. (2014) Flavin dependent monooxygenases. Arch Biochem Biophys $544: 2-17$

[5] Waldman AJ, Ng TL, Wang P et al. (2017) Heteroatomheteroatom bond formation in natural product biosynthesis. Chem Rev 117:5784-5863

[6] Hartmann M, Zeier T, Bernsdorff F et al. (2018) Flavin monooxygenase-generated N-hydroxypipecolic acid is a critical element of plant systemic immunity. Cell 173:456-469

[7] Heine T, Mehnert M, Schwabe R et al. (2017)

Thermochelin, a hydroxamate siderophore from Thermocrispum agreste DSM 44070. Solid State Phenom 262:501-504

[8] Andreadeli A, Platis D, Tishkov V et al. (2008) Structureguided alteration of coenzyme specificity of formate dehydrogenase by saturation mutagenesis to enable efficient utilization of NADP+. FEBS J 275:3859-3869

[9] Hoelsch K, Sührer I, Heusel M et al. (2013) Engineering of formate dehydrogenase: synergistic effect of mutations affecting cofactor specificity and chemical stability. Appl Microbiol Biotechnol 97:2473-2481

[10] Ngo TT, Brillhart KL, Davis RH et al. (1987) Spectrophotometric assay for ornithine decarboxylase. Anal Biochem 160:290-293

[11] Legaz M-E, Fontaniella B, de Armas R et al. (2001) Determination by high performance liquid chromatography of ornithine and lysine decaboxylases in sugar cane juices. Chromatographia 53:S260-S265

Funding: Open Access funding provided by Projekt DEAL.

Open Access: Dieser Artikel wird unter der Creative Commons Namensnennung 4.0 International Lizenz veröffentlicht, welche die Nutzung, Vervielfältigung erlaubt, sofern Sie den/die ursprünglichen Autor(en) und die Quelle ordnungsgemäß nennen, einen Link zur Creative Commons Lizenz beifügen und angeben, ob Änderungen vorgenommen wurden. Die in diesem Artikel enthaltenen Bilder und sonstiges Drittmaterial unterliegen ebenfalls der genannten Creative Commons Lizenz, sofern sich aus der Abbildungslegende nichts anderes ergibt. Sofern das betreffende Material nicht unter der genannten Creative Commons Lizenz steht und die betreffende Handlung nich nach gesetzlichen Vorschriften erlaubt ist, ist für die oben aufgeführt Weiterverwendungen des Materials die Einwilligung des jeweiligen Rechteinhabers einzuholen. Weitere Details zur Lizenz entnehmen Sie bitte der

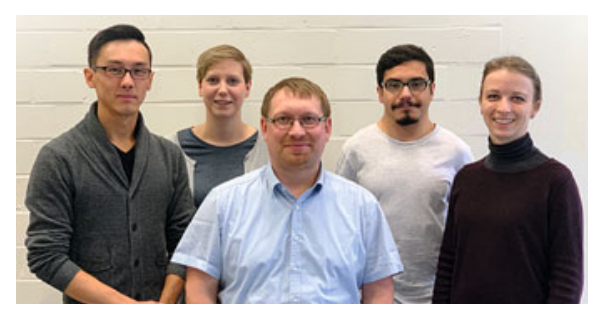

Artur Maier, Sarah Wansel, Dirk Tischler, Alvaro Gomez Baraibar und Carolin Mügge (v. I. n. r.).

Korrespondenzadresse:

Prof. Dr. Dirk Tischler

AG Mikrobielle Biotechnologie

Ruhr-Universität Bochum

Universitätsstraße 150

D-44780 Bochum

dirk.tischler@rub.de 